\title{
Experiments and Monte Carlo modeling of a higher resolution Cadmium Zinc Telluride detector for safeguards applications
}

\author{
Alessandro Borella \\ $S C K \cdot C E N$, Belgian Nuclear Research Centre, \\ Nuclear Science and Technology Studies unit, \\ Boeretang 200, Mol, B2400, Belgium \\ aborella@sckcen.be
}

Published 1 September 2016

\begin{abstract}
The Belgian Nuclear Research Centre is engaged in R\&D activity in the field of Non Destructive Analysis on nuclear materials, with focus on spent fuel characterization. A $500 \mathrm{~mm}^{3}$ Cadmium Zinc Telluride (CZT) with enhanced resolution was recently purchased. With a full width at half maximum of $1.3 \%$ at $662 \mathrm{keV}$, the detector is very promising in view of its use for applications such as determination of uranium enrichment and plutonium isotopic composition, as well as measurement on spent fuel. In this paper, I report about the work done with such a detector in terms of its characterization. The detector energy calibration, peak shape and efficiency were determined from experimental data. The data included measurements with calibrated sources, both in a bare and in a shielded environment. In addition, Monte Carlo calculations with the MCNPX code were carried out and benchmarked with experiments.
\end{abstract}

Keywords: Cadmium Zinc Telluride; CZT; Monte Carlo modeling; Detector Response; Non Destructive Assay; Gamma-ray spectrometry; Safeguards.

PACS numbers: 07.05.Tp, 07.85.Fv, 29.30.Kv, 29.40.-n, 29.40.Wk.

\section{Introduction}

Cadmium Zinc Telluride (CZT) detectors ${ }^{1}$ can be used for medium resolution gamma-ray spectrometry applications. The energy resolution at $662 \mathrm{keV}$, expressed in Full Width at Half Maximum (FWHM) for the full energy peak, can be as good as $1.3 \%,{ }^{2,3}$ and therefore one talks about medium resolution detectors, between the high resolution offered by High Purity Germanium $(0.2 \%)^{4}$ and the low resolution typical of e.g. NaI(Tl) detectors $(7 \%) .{ }^{4}$ Due to mechanisms related to the charge transport in the crystal, their peak shape exhibits a low energy tail. ${ }^{5,6}$ Since they do not require cooling, CZT detectors are portable and are used for example as an attribute tester for fresh and irradiated nuclear material during safeguards inspections. ${ }^{1,7}$ Limitations in crystal growth do not allow the production of large crystals, and therefore their efficiency is limited. For Safeguards application where a larger efficiency is required, measurement systems based on

This is an Open Access article published by World Scientific Publishing Company. It is distributed under the terms of the Creative Commons Attribution 3.0 (CC-BY) License. Further distribution of this work is permitted, provided the original work is properly cited. 
$\mathrm{LaBr}_{3}(\mathrm{Ce})$ scintillation detectors are preferred despite a worse $(\sim 2.8 \%$ FWHM at 662 $\mathrm{keV}$ ) energy resolution.

Considering their features, CZTs were chosen at SCK $• \mathrm{CEN}$ in view of developing a new measurement system for spent fuel elements, called the ForkBall ${ }^{8}$ detector. The CZT detector is used to measure the main gamma lines of fission products present in the spent fuel. The CZT data, together with the response of the other detectors present in the ForkBall, can then be used to verify the burnup of the spent fuel element. By carrying out measurements at different positions, an axial profile of the burnup can be determined. In addition, within the European SAfeguards Research and Development Association (ESARDA) NDA working group, ${ }^{9}$ an exercise to assess the capabilities of CZT and $\mathrm{LaBr}$ detectors to determine the $\mathrm{Pu}$ isotopic composition is being carried out.

Within this framework, the performances of a $500 \mathrm{~mm}^{3} \mathrm{CZT}$ detector were studied by measurements with calibrated point sources, including measurements in a shielded environment and in more complex geometries. The results obtained include optimal settings at high count rate for the data acquisition system that was used, effectiveness of the dead time correction, and peak shape parameters as a function of energy. An efficiency calibration for point sources was also determined and compared with the one determined with a Monte Carlo model. The model was also benchmarked with measurements with shielded sources and in more complex geometries. Having a reliable model of the detector is important in view of designing and optimizing the envisaged measurement system. The quoted uncertainties are one standard deviation.

\section{Measurements}

\subsection{Detectors and electronics setup}

A $10 \mathrm{~mm} \times 10 \mathrm{~mm} \times 5 \mathrm{~mm}$ quasi-hemispherical CZT detector (Model SDP500S from Ritec Ltd. $)^{2}$ with a preamplifier embedded in the detector head was used. This detector was coupled with the MCA52 $7^{10}$ module from GBS Elektronik GmbH. This module supplies the high voltage to the detector and power to the pre-amplifier. In addition, it digitally processes the signal obtained after the shaping of the input signal provided by the preamplifier, and spectra can be acquired with the WinSPEC software package. ${ }^{11}$

The MCA settings used were recommended by the manufacturer ${ }^{12}$ in high count rate applications such as the ones that are envisaged in case of spent fuel measurements; these settings include a LLD of 0, Trigger Level of 7, Energy Level of 0.5\%, FlatTop time of $0.7 \mu \mathrm{s}$, a Shaping Time of $0.7 \mu \mathrm{s}$, and Trigger Filter $[+1 ;-2 ;+1]$ for digital processing of the signal. The spectra were acquired in 2048 channels. The pole zero compensation (PZC) was checked before each measurement. Unless indicated otherwise for the determination of the net peak areas, we used WinSPEC; the net peak areas were obtained by summing the counts in the peak region of interest (ROI) and assuming a linear behaviour for the background, which was averaged over 3 channels before and after the ROI. 


\subsection{Measurements with point bare sources}

Measurements with ${ }^{152} \mathrm{Eu},{ }^{60} \mathrm{Co}$, and ${ }^{137} \mathrm{Cs}$ bare point sources were carried out to verify the dead time correction and optimal settings for MCA527, and to determine the energy calibration and the peak shape parameters as a function of energy. In addition, the efficiency calibration for point sources was also determined.

The sources used are listed in Table 1 . Since the ${ }^{60} \mathrm{Co}$ and ${ }^{137} \mathrm{Cs}$ sources are calibrated in air kerma, the uncertainty on the activity for these sources is a combination of the uncertainty on the declared air kerma value and the conversion coefficient, ${ }^{13}$ which was set to $2.0 \%$.

Table 1. Calibrations sources used.

\begin{tabular}{cccc}
\hline Nuclide & Name & Activity / MBq & Uncertainty \\
\hline${ }^{152} \mathrm{Eu}$ & MOL5 & 24.755 & $2.4 \%$ \\
${ }^{60} \mathrm{Co}$ & $\mathrm{P} 3$ & 366.912 & $3.0 \%$ \\
${ }^{60} \mathrm{Co}$ & $\mathrm{P} 4$ & 2818.4 & $3.0 \%$ \\
${ }^{137} \mathrm{Cs}$ & $\mathrm{P} 11$ & 4985.1 & $3.7 \%$ \\
${ }^{137} \mathrm{Cs}$ & $\mathrm{P} 12$ & 106002 & $3.5 \%$ \\
\hline
\end{tabular}

\subsubsection{Energy and peak shape calibration}

A linear equation was used for the energy calibration; the obtained gain was very stable over several months of operation with a typical value of $1.04 \pm 0.01 \mathrm{keV} / \mathrm{ch}$. The offset was on the order of few $\mathrm{keV}$.

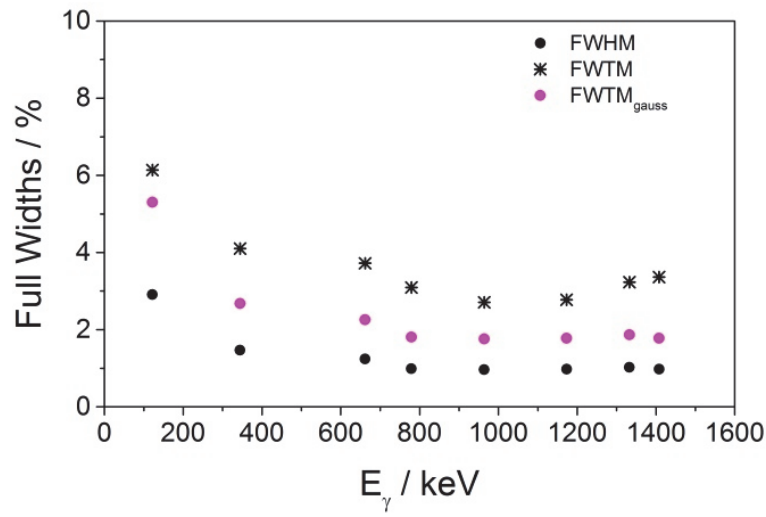

Fig. 1. Peak shape parameters FWHM, FWHM gauss and FWHM for the considered CZT detector.

To study the peak shape of the CZT detector in use, the FWHM and Full-Width-atTenth-Maximum (FWTM) were determined. The obtained results are shown in Fig. 1. The FWTM that would be expected in an ideal Gaussian peak is also reported (FWTM gauss). This number has been obtained by multiplying FWHM by 1.8226 , which is 
the ratio between FWTM and FWHM for a Gaussian peak. The deviation of FWTM from FWTM $_{\text {gauss }}$ is an indication of the impact of the low energy tail.

\subsubsection{Dead time correction}

To verify the correctness of the proposed dead time correction when carrying out measurements with MCA527 and using WinSPEC as the data acquisition program, the so-called "two sources" method ${ }^{14}$ was used. Different measurements are carried out where one source is kept at a fixed distance from the detector; in each measurement a second source is kept at a distance that varies during different measurements. The principle of this method is that the dead time corrected count rate for the fixed source should always be the same, independent of the total count rate. To limit interferences, the fixed source should have much higher gamma-ray energy than the variable source.

The P4 ${ }^{60}$ Co source was the high energy fixed source at $320 \mathrm{~cm}$ from the detector. A ${ }^{137} \mathrm{Cs}$ source was positioned at different distances from the detector to obtain different total count rates. Despite the use of a pile-up rejection algorithm, a pile-up peak at about $1324 \mathrm{keV}$ could be seen from about $30 \mathrm{kcps}$. Due to the resolution of about $13 \mathrm{keV}$, this peak overlaps with the $1332 \mathrm{keV}$ from ${ }^{60} \mathrm{Co}$. To estimate this contribution, separate measurements with the ${ }^{137} \mathrm{Cs}$ source only were carried out. These spectra with ${ }^{137} \mathrm{Cs}$ only were then subtracted to account for the pile-up in the measurements with ${ }^{137} \mathrm{Cs}$ and ${ }^{60} \mathrm{Co}$.

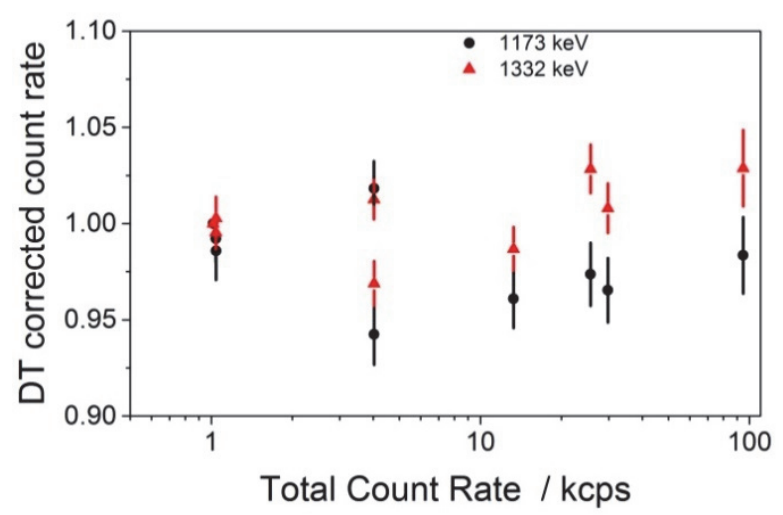

Fig. 2. The dead time corrected net count rates for the $1173 \mathrm{keV}$ and $1332 \mathrm{keV}$ gamma-rays as a function of the total count rate. The count rates are relative to the reference case without the ${ }^{137} \mathrm{Cs}$ source.

The measurement times were about 30 minutes. The dead time was determined by the MCA527 relative to the real measurement time. The dead time corrected net count rates for the $1173 \mathrm{keV}$ and $1332 \mathrm{keV}$ lines relative to the reference case without the ${ }^{137} \mathrm{Cs}$ source are given in Fig. 2. The net peak area was determined by summing the counts in the ROI and assuming a linear behaviour for the background, which was averaged over 3 channels before and after the ROI. The data for the $1332 \mathrm{keV}$ peak exhibit a lower scatter in the data when compared with the data from the $1173 \mathrm{keV}$ peak, which is possibly 
affected by the Compton shoulder of the $1332 \mathrm{keV}$ peak. The data reveal that the dead time correction is valid within the uncertainties up to a count rate of about $90 \mathrm{kcps}$ with a corresponding dead time of about $35 \%$.

Measurements with different values of the MCA527 settings were also done, and it was noticed that different settings for LLD, Energy threshold, Trigger filter and Trigger level may introduce a bias in the dead time corrections up to $10 \%$. These results stress the importance of having correct MCA527 settings to obtain a reliable value for the dead time correction.

\subsubsection{Efficiency measurements}

The measurements with the sources listed in Table 1 were also used to determine the total photo-peak detection probability (or full energy peak efficiency) of the detector. The efficiency $\varepsilon$ was calculated as given in Eq. (1):

$$
\epsilon=\frac{C_{i}}{\beta_{i} A},
$$

where $C_{i}$ is the is net peak area count rate for the gamma-ray $i, A$ is the activity of the source, and $\beta_{i}$ is the branching for the gamma-ray $i$. Measurement data at different distances were scaled with a factor $1 / r^{2}$ where $r$ is the distance source-detector in meters; the efficiency is then obtained at $1 \mathrm{~m}$ distance from the source and is shown in Fig. 3. This scaling is valid only if the distance is much larger than the detector dimensions.

The gamma-ray energies of ${ }^{152} \mathrm{Eu}$ (at 121.8, 244.7, 778.9, 964.1 and $1408.0 \mathrm{keV}$ ), of ${ }^{60} \mathrm{Co}$ (at 1173.2 and $1332.5, \mathrm{keV}$ ), and of ${ }^{137} \mathrm{Cs}$ (at $661.7 \mathrm{keV}$ ) were considered. For some energies, more than one data point is available. Only the statistical uncertainty associated with the determination of the net peak area and the source activity were considered in this analysis.

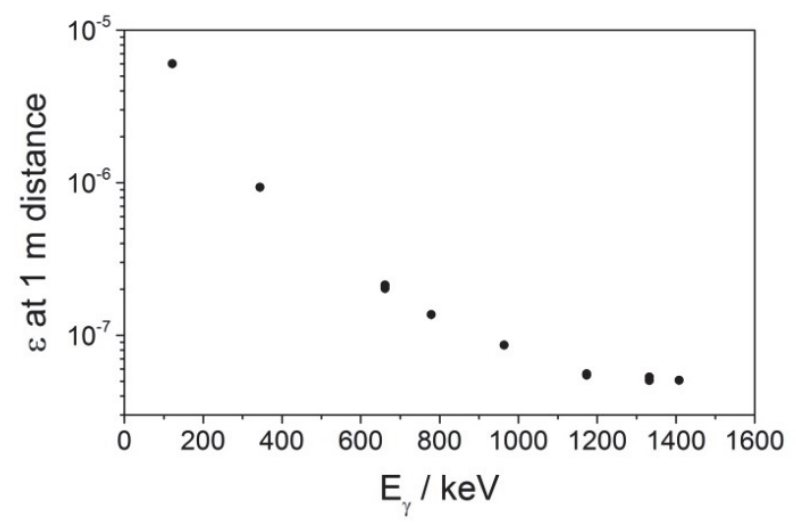

Fig. 3. Measured efficiency at $1 \mathrm{~m}$ distance as a function of gamma-ray energy. 


\subsection{Measurements with complex geometries}

\subsubsection{Measurements with shielded sources}

In addition to measurements with bare sources, measurements with $\mathrm{Pb}$ disks with a diameter of $27 \mathrm{~mm}$ were also carried out. The CZT detector was placed inside a $\mathrm{Pb}$ structure with a cylindrical cavity where the $\mathrm{Pb}$ disks were placed in front of the detector as shown in Fig. 4(A). The detector was positioned at $150 \mathrm{~cm}$ from the P11 ${ }^{137} \mathrm{Cs}$ source. The thickness of the disks ranged between $1 \mathrm{~mm}$ and $16 \mathrm{~mm}$, and the disks were combined to obtain shields of different thicknesses. The obtained net count rate as a function of the combined thickness was determined and is shown in Table 2.

\subsubsection{Vertical scan measurements}

The CZT detector was placed inside a $\mathrm{Pb}$ structure with a cylindrical cavity as shown in in Fig. 4(B). By changing the vertical position of the source, the detector response varies due to the different solid angle, the different distance, and the different shadow effect of the $\mathrm{Pb}$. This situation is similar to the one that should be present in the envisaged measurement geometry for spent fuel measurements. Therefore, understanding the behavior of the detector in such a situation and the ability to model and predict such behavior are of importance.

The P11 ${ }^{137} \mathrm{Cs}$ source at $150 \mathrm{~cm}$ distance was kept fixed during these measurements, while the height of the $\mathrm{Pb}$ castle was changed. The accuracy of the quoted vertical position was estimated to be $0.5 \mathrm{~cm}$.
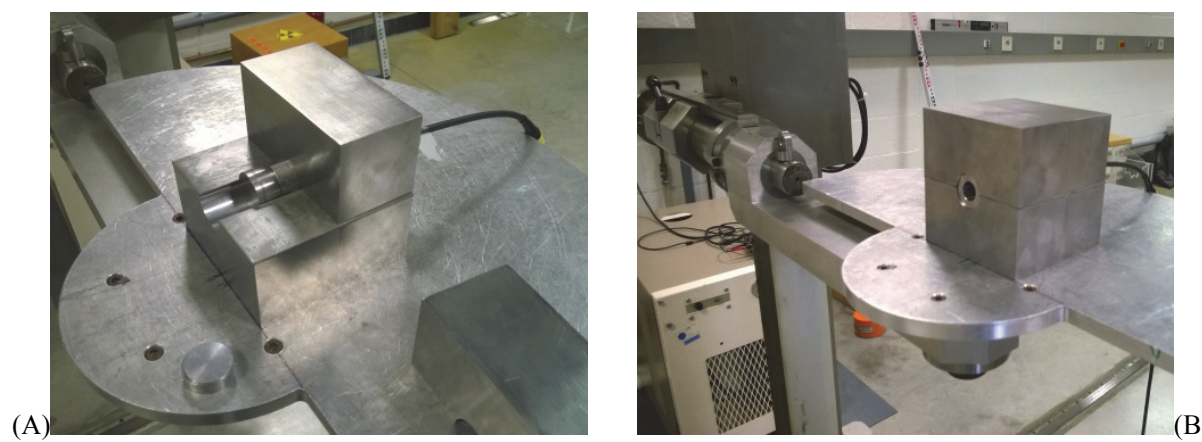

Fig. 4. Shielded sources (A) and vertical scan (B) measurement setup.

\section{Monte Carlo Modelling and Benchmark}

\subsection{Efficiency}

The measured full energy peak efficiencies were compared with the ones that were obtained with a Monte Carlo model of the measurement setup by using the code MCNPX, ${ }^{15}$ version 2.7. The CZT crystal and casing, based on the technical description 
made available by the manufacturer, ${ }^{2,3}$ were included in the model. The point sources were supposed to be unshielded and isotropic at the measurement distance.

The full energy peak efficiency was determined with an energy deposition (F8) tally. To reduce the computational time, the gamma-rays were emitted only in a limited cone centered on the detector center covering a region of at least $4 \mathrm{~cm}$. The data were then corrected for the solid angle used, and the efficiency for an isotropic source was then determined. The ratio between calculated efficiency and experimental efficiency is shown in Fig. 5. The quoted uncertainties are a combination of the statistical uncertainty associated with the measured data, the uncertainty associated with Monte Carlo simulations, and the uncertainty on the activity of the source, which is often the dominating component.

The data in Fig. 5 indicate that the efficiency computed with Monte Carlo methods is overestimated. This can be explained by the fact that the effective volume of the detector is smaller than the nominal $500 \mathrm{~mm}^{3}$. In addition, the non-uniform electric field ${ }^{5}$ and the fact that the detection probability is a function of the position inside the detector ${ }^{5,6,16,17}$ cannot be accounted for in a straightforward way in the simulations. These phenomena affect the observed deposited energy in the detector and explain the observed peak shape. However, based on the indications of Ref. 18, they should not impact the efficiency. The trend with the gamma-ray energy up to $\sim 1200 \mathrm{keV}$ can be explained by the presence of dead zones in the CZT crystal or regions where the electric field is not intense enough to ensure complete charge collection.

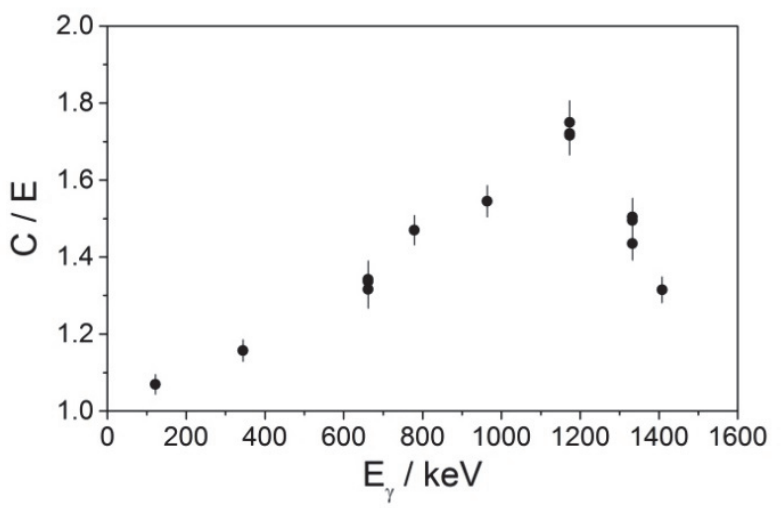

Fig. 5. Ratio between calculated (C) and measured (E) efficiency as a function of gamma-ray energy.

Given the general tendency of any efficiency curve in gamma-ray spectrometry to decrease with increasing energy, the $1332 \mathrm{keV}$ peak area should always be substantially lower than the 1173 peak area. However, our results do not always exhibit this characteristic. The net peak areas of the two peaks are very similar, and consequently, the $\mathrm{C} / \mathrm{E}$ at $1332 \mathrm{keV}$ is lower that the $\mathrm{C} / \mathrm{E}$ at $1173 \mathrm{keV}$. This behavior is not expected and can be due to inaccuracies in the algorithm used for the determination of the net peak area. 
For the ${ }^{152} \mathrm{Eu}$ data, the MOL5 source was used. We remark the anomalous behavior of the $\mathrm{C} / \mathrm{E}$ value at $1408 \mathrm{keV}$; again this could be ascribed to a wrong determination of the net peak area for this peak, which exhibits a very long tail. However, the data for the $1408 \mathrm{keV}$ peak are consistent with the one of the $1332 \mathrm{keV}$ peak indicating a change in the $\mathrm{C} / \mathrm{E}$ trend as a function of gamma-ray energy.

\subsection{Complex geometries}

\subsubsection{Shielded sources}

The measurement geometry was modeled in MCNPX, using the measured weight to determine the effective thickness with the nominal density of the shielding material being $11.34 \mathrm{~g} / \mathrm{cm}^{3}$ for $\mathrm{Pb}$.

The transmission factors for the ${ }^{137} \mathrm{Cs} 662 \mathrm{keV}$ line, measured and estimated with a Monte Carlo model, are given in Table 2 as a function of the thickness of the disks. The agreement between the model and the experimental results is good, although the modeled data seem slightly underestimated as the thickness of the disk increases. This may be due to the presence of $\mathrm{Sb}$ in $\mathrm{Pb}$, slightly increasing the transmission of the disks.

The obtained results confirm the reliability of the developed models. The ratio between measured and calculated count rate in the absence of $\mathrm{Pb}$ was $1.35 \pm 0.05$, in good agreement with the values given in the previous section for the $\mathrm{C} / \mathrm{E}$ ratio on the efficiency of the ${ }^{137} \mathrm{Cs}$ gamma-ray energy. The uncertainty includes the uncertainty on the activity of the source.

Table 2. Peak count rates and transmission factors as a function of the shielding thickness.

\begin{tabular}{cccc}
\hline Effective thickness / mm & Net Peak count rate / cps & Measured Transmission & Modelled Transmission \\
\hline 0.00 & $371.8 \pm 1.8$ & 1.000 & 1.000 \\
0.95 & $333.0 \pm 1.6$ & $0.896 \pm 0.006$ & $0.898 \pm 0.006$ \\
1.92 & $299.3 \pm 1.6$ & $0.805 \pm 0.006$ & $0.800 \pm 0.005$ \\
2.87 & $265.5 \pm 1.5$ & $0.714 \pm 0.005$ & $0.715 \pm 0.005$ \\
4.82 & $216.6 \pm 1.4$ & $0.583 \pm 0.005$ & $0.568 \pm 0.004$ \\
6.74 & $172.1 \pm 1.3$ & $0.463 \pm 0.004$ & $0.450 \pm 0.004$ \\
7.75 & $159.0 \pm 1.1$ & $0.427 \pm 0.004$ & $0.401 \pm 0.003$ \\
10.63 & $115.3 \pm 1.0$ & $0.310 \pm 0.003$ & $0.287 \pm 0.003$ \\
14.50 & $73.4 \pm 0.6$ & $0.197 \pm 0.002$ & $0.183 \pm 0.002$ \\
20.36 & $38.1 \pm 0.4$ & $0.103 \pm 0.001$ & $0.092 \pm 0.001$ \\
26.17 & $19.9 \pm 0.2$ & $0.053 \pm 0.001$ & $0.046 \pm 0.001$ \\
\hline
\end{tabular}

\subsubsection{Vertical scan}

For the vertical scan, the measurement geometry was also modeled in MCNPX using the available design information. Both the calculated and measured count rates were normalized to the vertical position of $0 \mathrm{~cm}$. The ratio between measured and calculated 
count rate in the horizontal position was $1.32 \pm 0.06$, in good agreement with the values given in the previous sections for the ${ }^{137} \mathrm{Cs}$ gamma-ray energy. The uncertainty includes the uncertainty on the activity of the source.

A comparison between experimental and calculated data is given in Table 3. The experimental and simulated data are consistent with each other within the quoted uncertainties. The uncertainty on the $\mathrm{C} / \mathrm{E}$ ratio includes the uncertainty on the experimental data and simulations but does not account for the uncertainty on the activity of the source.

Table 3. Net peak count rate as a function of the vertical position and $\mathrm{C} / \mathrm{E}$ ratio.

\begin{tabular}{ccc}
\hline Vertical Position / cm & Net Peak Count rate / cps & C/E \\
\hline $0.0 \pm 0.5$ & $375.7 \pm 1.0$ & $1.000 \pm 0.025$ \\
$1.0 \pm 0.5$ & $376.4 \pm 1.0$ & $1.002 \pm 0.025$ \\
$2.0 \pm 0.5$ & $374.3 \pm 1.0$ & $0.976 \pm 0.025$ \\
$3.0 \pm 0.5$ & $375.8 \pm 1.6$ & $1.015 \pm 0.026$ \\
$4.0 \pm 0.5$ & $372.5 \pm 1.7$ & $1.036 \pm 0.026$ \\
$5.0 \pm 0.5$ & $372.1 \pm 1.7$ & $0.982 \pm 0.026$ \\
$8.0 \pm 0.5$ & $362.7 \pm 1.7$ & $1.015 \pm 0.026$ \\
$11.0 \pm 0.5$ & $341.4 \pm 1.5$ & $1.000 \pm 0.027$ \\
$14.0 \pm 0.5$ & $318.6 \pm 1.5$ & $1.015 \pm 0.028$ \\
$17.0 \pm 0.5$ & $287.5 \pm 1.3$ & $1.051 \pm 0.030$ \\
\hline
\end{tabular}

\section{Conclusions}

The results of measurements with a $500 \mathrm{~mm}^{3} \mathrm{CZT}$ detector coupled with the MCA527 signal processing unit and the WinSPEC data acquisition software were presented. The experimental data revealed that with the settings used, the dead time correction is reliable up to almost $100 \mathrm{kcps}$. In addition, measurement with bare point sources allowed the determination of the detector energy calibration, peak shape, and efficiency. Measurements with more complex geometries, such as shielded sources and vertical scan measurements of a collimated system, were also carried out with a ${ }^{137} \mathrm{Cs}$ source.

The results of the experiments were used to benchmark developed Monte Carlo models. A comparison between measurements and simulations indicates that the model systematically overestimates the efficiency. The comparison between simulations and measurements in all the geometries considered for the ${ }^{137} \mathrm{Cs}$ sources gave consistent results. The overestimation of the efficiency can be due to an overestimation of the active volume of the detector. This explanation is consistent with the observed trend in the overestimation as a function of the gamma-ray energy up to $\sim 1200 \mathrm{keV}$. After $1200 \mathrm{keV}$, an inversion of the trend was observed that could not yet be explained in a satisfactory way. Future work will aim at improving both the Monte Carlo model of the detector and the algorithm used for the determination of net peak areas. 


\section{Acknowledgments}

The author would like to thank Dr. Michel Bruggeman, Mr. Jörg Brutscher and Mr. Victor Ivanov for their advice and helpful support; a special thanks to Mrs. Anete Korpa for the careful reading of the manuscript.

\section{References}

1. R. Arlt, V. Ivanov, K. Parnham, and K. Advantages and use of CdZnTe detectors in safeguards measurements, In Proceedings of the MPA\& C Conference, Obninsk, Russia (2000).

2. Spectrometric Detection Probe with Large Volume CdZnTe Detector SDP 500 (S), Operator's manual, Ritec (2013).

3. V. Ivanov, private communication.

4. P. A. Russo and D. T. Vo, Gamma-Ray Detectors For Nondestructive Analysis, Los Alamos Report, LA-UR-05-3813 (2005).

5. E. Kalemci, J.L. Matteson, R.T. Skelton, P.L. Hink, and K.R. Slavis, Model calculations of the response of CZT strip detectors, in Proc. SPIE 3768, Hard X-Ray, Gamma-Ray, and Neutron Detector Physics, 360 (1999); doi:10.1117/12.366601.

6. Y.X. Dardenne, T.F. Wang, A.D. Lavietes, G.J. Mauger, W.D. Ruhter, and S.A. Kreek, Nucl. Instr. Meth. Phys. Res. A422, 159 (1999), http://dx.doi.org/10.1016/S0168-9002(98)00947-4.

7. Safeguards techniques and equipment, International Atomic Energy Agency, ISBN 978-92-0118910-3 (2011).

8. A. Borella, G. Bergmans, K. van der Meer, R. Delporte, and F. Flachet, Advances in the Development of a Spent Fuel Measurement Device in Belgian Nuclear Power Plants, In Symposium on International Safeguards, Vienna, Austria, International Atomic Energy Agency (2014), p. 272.

9. https://esarda.jrc.ec.europa.eu/index.php?option=com_content\&view $=$ article $\&$ id $=62 \&$ Itemid $=1$ 23.

10. MCA-527, Digital Multi-Channel Analyzer, GBS-Elektronic, User Manual (2012).

11. WinSPEC for Inspectors, GBS-elektronik, http://www.gbs-elektronik.de/en/downloads/sortedby-category/software/.

12. Jörg Brutscher, private communication (2013).

13. H. Wasserman and W. Groenewald, European J. Nucl. Med. 14, 569 (1988).

14. R. Berndt and P. Mortreau, Performance test of the Multi-Channel Analyzer MCA-527 for Nuclear Safeguards Application, Report EUR 26165 EN, 2013.

15. MCNPX User's Manual, Version 2.7.0, D. B. Pelowitz, ed., Los Alamos National Laboratory report LA-CP-11-00438 (April 2011).

16. S.D. Chun, S.H. Park, J.H. Ha and H.S. Kim, Monte Carlo simulation of a CZT detector, in Proc. $4^{\text {th }}$ Int. Symposium on Radiation Safety and Detection Technology, J. Nucl. Sci. Tech., Suppl. 5 (2008), pp. 549-555.

17. L. Chen and Y.X. Wei, Appl. Radiat. Isot. (2008) doi: 10.1016/j.apradiso.2008.01.008.

18. J.M. Perez, Z. He, D.K. Wehe, and Y.F. Du, IEEE Trans. Nucl. Sci. 49, 2010 (2002), doi: 10.1109/TNS.2002.801512. 